\title{
CHEMISTRY OF NOVA ENVELOPES
}

\author{
A.A. Boyarchuk, L.I. Antipova \\ Astronomical Council of the USSR Academy of Science
}

Novae form a very interesting class of non-stationary objects. A considerable amount of observational data, both photometric and spectroscopic, covering different stages of nova outbursts, have been stored up by observers for many years. Processing and systematization of these data has lead to a better understanding of the physical processes taking place in the nova outbursts.

The first spectra of novae at maximum brightness proved to be similar to the spectra of supergiants; their distinctive feature was the presence of the strong lines of lines from $C, N$ and $O$ (Mclaughlin, 1936). This fact lead to suspect that these elements must be overabundant in the nova envelopes.

The first quantitative analysis of a nova spectrum was made by Mustel and Boyarchuk (1959) on the basis of spectrograms of DQ Her obtained during its maximum brightness. An analysis of the absorption lines by the curve-of-growth method proved the abundances of light elements ( $C, N$ and $O$ ) in the nova envelope to really exceed the "normal" values by roughly a factor of 100 (Mustel and Boyarchuk 1959). Since that time these spectrograms have been revised several times (Mustel and Boyarchuk 1959a; Mustel and Baranova 1965), but the main conclusion about the large overabundances of $\mathrm{C}, \mathrm{N}$ and $\mathrm{O}$ remained practically the same. Pottasch $(1959,1957)$ was the first to apply a technique based on the analysis of the emission lines, originally developed for planetary nebulae, to estimatee the chemical composition from the spectra of six classical novae and the recurrent nova RS Oph in its nebular phase.

The discovery of the binarity of novae increased the interest in such objects. It became clear that the reason of a nova outburst should be looked for in their binarity. According to the generally accepted model, all novae are close binary systems, and their outbursts occur in a layer of matter accreted on the surface of a white dwarf, lost by a cooler companion. This matter is rich in hydrogen - a potential nuclear fuel. Hydrodynamical analyses of nova outbursts within the framework of this model (Starrfield et al. 1972) prove that the chemical composition of the accreted matter and, in particular, of the layer where the outburst begins, plays rather an important role in producing. Therefore the analysis of the chemical composition of novae became of special importance. It is considered that nova outbursts may also introduce a contribution to the enrichment of the Galaxy's matter with some elements and isotopes.

There are two ways to study the chemical composition of the envelopes ejected by novae: (a) from the analysis of the absorption lines and (b) from the analysis of emission lines. In most investigations the latter approach was used. There is a number of papers devoted to the critical analysis and to the comparison of these two methods (see, for example, Collin-Souffrin 1977; Williams 1977). The development of space research permitted to study the chemical composition of nova sheils from ultraviolet and infrared data. The 
Galactic novae in outburst

\begin{tabular}{|c|c|c|c|c|c|c|c|c|c|c|c|}
\hline & \multirow{2}{*}{ Novae } & \multirow{2}{*}{$\begin{array}{c}\text { Outburst } \\
\text { year }\end{array}$} & \multicolumn{3}{|c|}{ R.A. } & \multicolumn{3}{|c|}{ Deo. } & \multirow{2}{*}{$m_{\max }$} & \multirow{2}{*}{$\begin{array}{c}\text { Ampli- } \\
\text { tude }\end{array}$} & \\
\hline 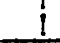 & & & $h r$ & $\mathrm{mn}$ & sec! & deg & $\mathrm{mn}$ & sec! & & & \\
\hline I. & V1500 Cyg & I975 & $2 I^{h}$ & $09^{m}$ & $53^{\prime}$ & $47^{\circ}$ & $56^{\prime}$ & $4 I^{\prime \prime}$ & $2^{m}, 22$ & $2 I^{m}$ & $39^{d}$ \\
\hline 2. & $R R$ P1c & I925 & 6 & 35 & IO & -62 & 35 & 47 & $I, 2$ & $I 2,8$ & I50 \\
\hline 3. & $\mathrm{~V} 603 \mathrm{Aql}$ & I9I8 & I8 & 46 & $2 I$ & $\infty$ & $3 I$ & 00 & $-I, I$ & $\mathrm{I} 2,0$ & 8 \\
\hline 4. & CP IaO & I936 & 22 & 12 & 00 & 55 & 07 & 06 & $2, I$ & I5, 6 & IO \\
\hline 5. & GK Per & I90I & 3 & 27 & 48 & 43 & 44 & 04 & 0,2 & $I 4,0$ & I3 \\
\hline 6. & CP Pup & I942 & 8 & 07 & 58 & -35 & 09 & & 0,5 & 17,0 & 7 \\
\hline 7. & IV Cep & I97I & 22 & 00 & 57 & 53 & OI & I8 & 7,5 & I7, 5 & 37 \\
\hline 8. & HR Del & 1967 & 20 & 40 & 00 & I8 & 59 & 00 & 3,5 & $\mathrm{I} 2,5$ & 230 \\
\hline 9. & DQ Her & I934 & I8 & 06 & 05 & 45 & $5 I$ & OI & $\mathrm{I}, 3$ & 14,8 & 100 \\
\hline 10. & GQ Mus & I983 & II & 49 & 35 & -66 & 55 & 43 & 6,5 & $I 4,5$ & 40 \\
\hline II. & V533 Her & 1963 & I8 & I2 & 46 & $4 I$ & 50 & $2 I$ & 3,0 & $I 4,9$ & 44 \\
\hline I2. & T Aur & I89I & 5 & 28 & 46 & 30 & 24 & 35 & $4, I$ & I5, 8 & 80 \\
\hline I3. & $R R T \in I$ & 1949 & 20 & 00 & 18 & -55 & 52 & 00 & 6,8 & & 1820 \\
\hline I4. & V1668 Cyg & 1978 & $2 I$ & 40 & 38 & 43 & 48 & 00 & 6,5 & I4 & 23 \\
\hline I5. & V693 CrA & I98I & 18 & 38 & 30 & -37 & 34 & 59 & 7. & I4 & I5 \\
\hline I6. & V1370 AqI & I982 & I9 & 20 & 50 & 02 & 24 & 00 & 6,5 & $I 3,0$ & I7 \\
\hline I7. & LV VuI & 1968 & 19 & 45 & 57 & 27 & 02 & 48 & 5,7 & I6,9 & 35 \\
\hline I8. & DK Lac & I950 & 22 & 45 & 35 & 52 & 45 & 36 & 5,0 & $\mathrm{I} 5,5$ & 29 \\
\hline
\end{tabular}


analysis of all the data, available by now, on the chemical composition of novae shells obtained by different methods and in different spectral regions is of interest.

By now, we have information about the chemical composition of the envelopes of about twenty classical novae (see Table 1). These novae belong to different velocity classes, amplitudes, etc. Table 2 contains the determinations of the chemical composition for these novae. Column 4 indicates which type of lines have been analyged. Whenever several determinations of the chemical abundances were available, the results are given in chronological order; the time (days or years) from the moment of maximum brightness (0d) to the moment when the analysed spectrograms were obtained (negative values mean that pre-maximum spectra were analysed) are given in Column 3 . The spectral region under analysis is indicated in Column 5 . In all cases, except those mentioned in the remarks, abundances relative to hydrogen are given (lg $\mathrm{N}(\mathrm{H})=12)$.

One can see from Table 2 that many of the investigations on the chemical composition were based on the analysis of the emission lines during nebular or earlier stages. For four novae the chemical composition was determined by using absorption lines; for five ones it was based on spectra in the ultraviolet region. The relative abundances of chemical elements $([X]=1 \mathrm{~g}(\mathrm{X}) \mathrm{sova}-\lg (\mathrm{X}) \mathrm{gun})$, calculated on the basis of Table 2 , are shown in Fig. 1. It can be seen from Fig. 1 that the general properties of the envelopes of all the novae are: 1) an abnormally high abundance of $N$ and $O$; 2) He-content higher than "normal"; 3) a high abundance of Ne in some novae. Besides, one should note here that overabundances of intermediate mass elements ( $\mathrm{Na}, \mathrm{Mg}, \mathrm{Al}, \mathrm{Si}, \mathrm{S}$ ) were found in the envelopes of two novae - V693 CrA and V1370 Aql - named "neon" novae. Let us analyse in detail each of these anomalies.

CNO-abundances. One can see from Table 2 and Fig. 1 that the abundances of $\mathrm{N}$ and $O$, and in some cases of $C$, exceed the "normal" values by the order of $1-2$ dex on the average. Unfortunately, the data in Table 2 are very inhomogeneous, therefore comparisons are difficult. The reasons for these inhomogeneities are: 1) various investigations were based on spectra taken at different resolutions; 2) the methods of analysis also differ and, in particular, the methods to determine $T_{e}$ and $\left.N_{e} ; 3\right)$ the emission lines chosen to determine the abundances in various investigations often do not coincide. And finally, the outburst stage, which has to do with investigation, is of great importance. During the initial period of decrease in brightness, the hydrogen Balmer emission lines that, as a rule, are used in the analysis of the chemical composition (usually $H \propto$ or $H_{\beta}$ ) are often distorted by self-absorption. As shown by Ferland (1978), for the extremely fast nova V1500 Cyg, the case $B$ conditions for Balmer emission were achieved in 100 days. This period is much longer for other novae. In many investigation self-absorption in Balmer lines has not been taken into account. There are very few papers where the presence of self-absorption was checked for, and if necessary, corrections were made. Self absorption, to the extent it is not taken into account, distorts the abundances of chemical elements relative to hydrogen. Despite the data inhomogeneity, one may try to make an analysis of the abundances of the $C, N$ and $O$ in the novae envelopes.

According to the predictions of thermonuclear runaway models (Starrfield et al. 1977, 1978) the main characteristics of an outburst are determined by the number of CNO-nuclei at the bottom of the 


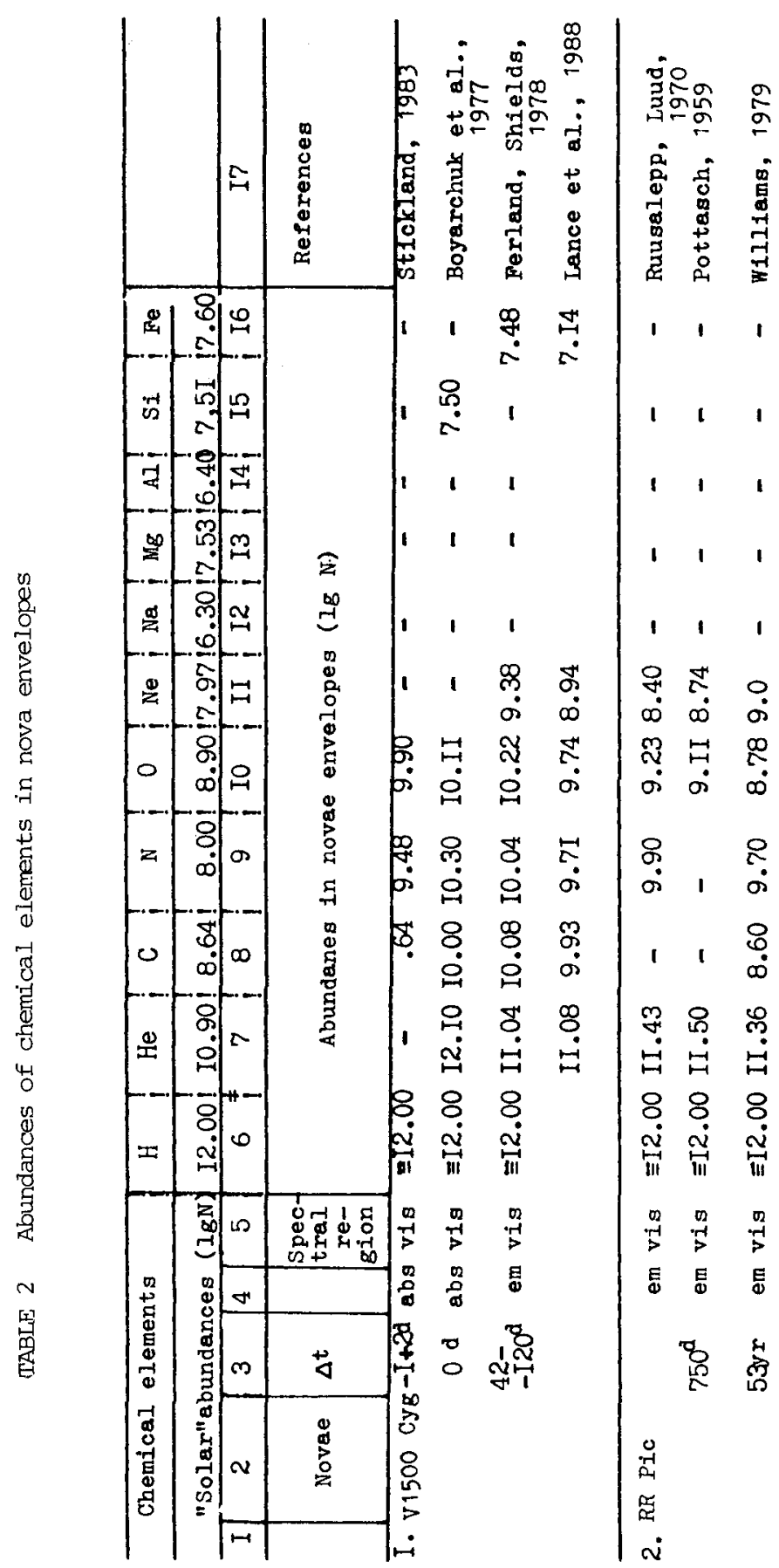




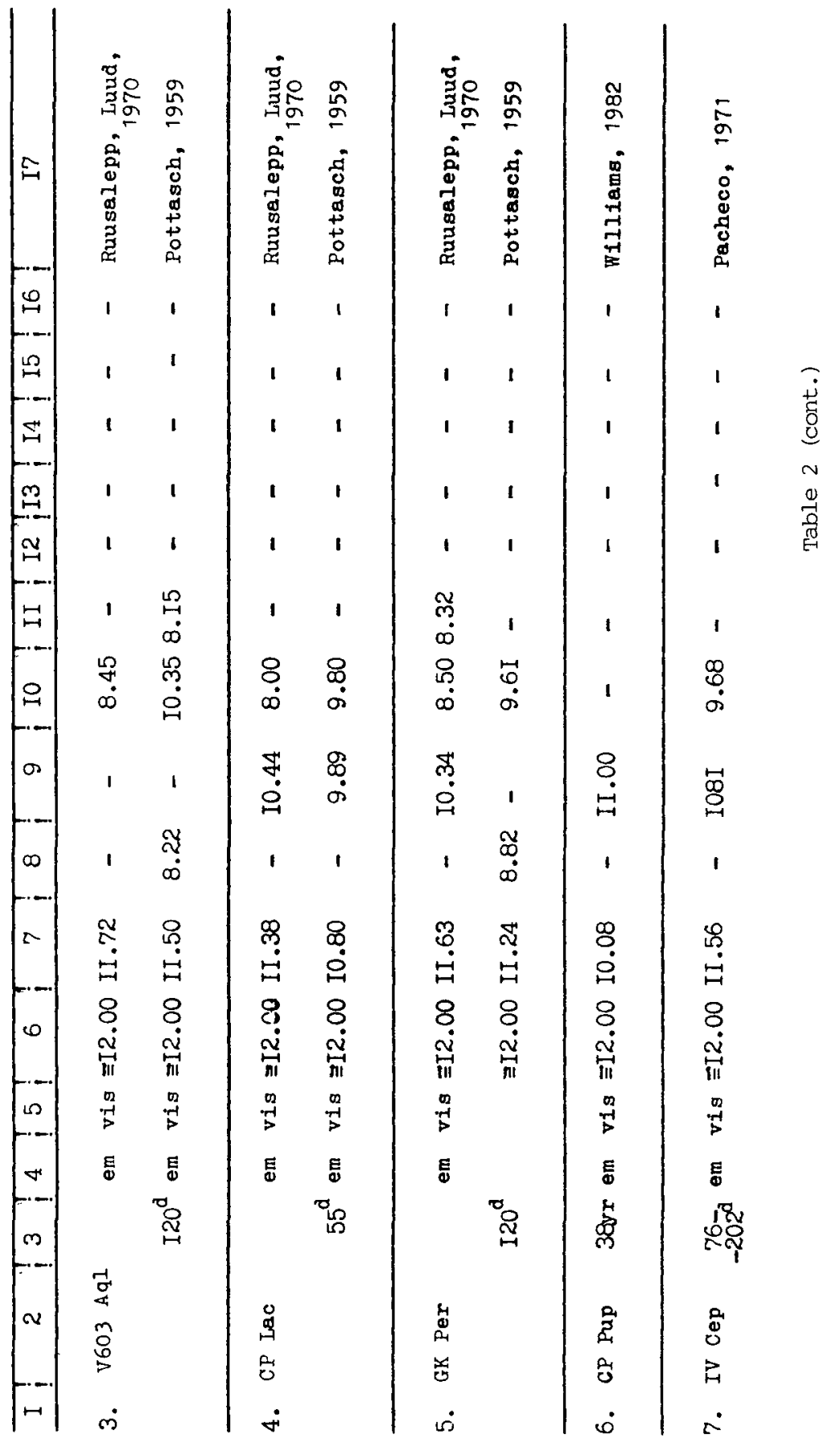




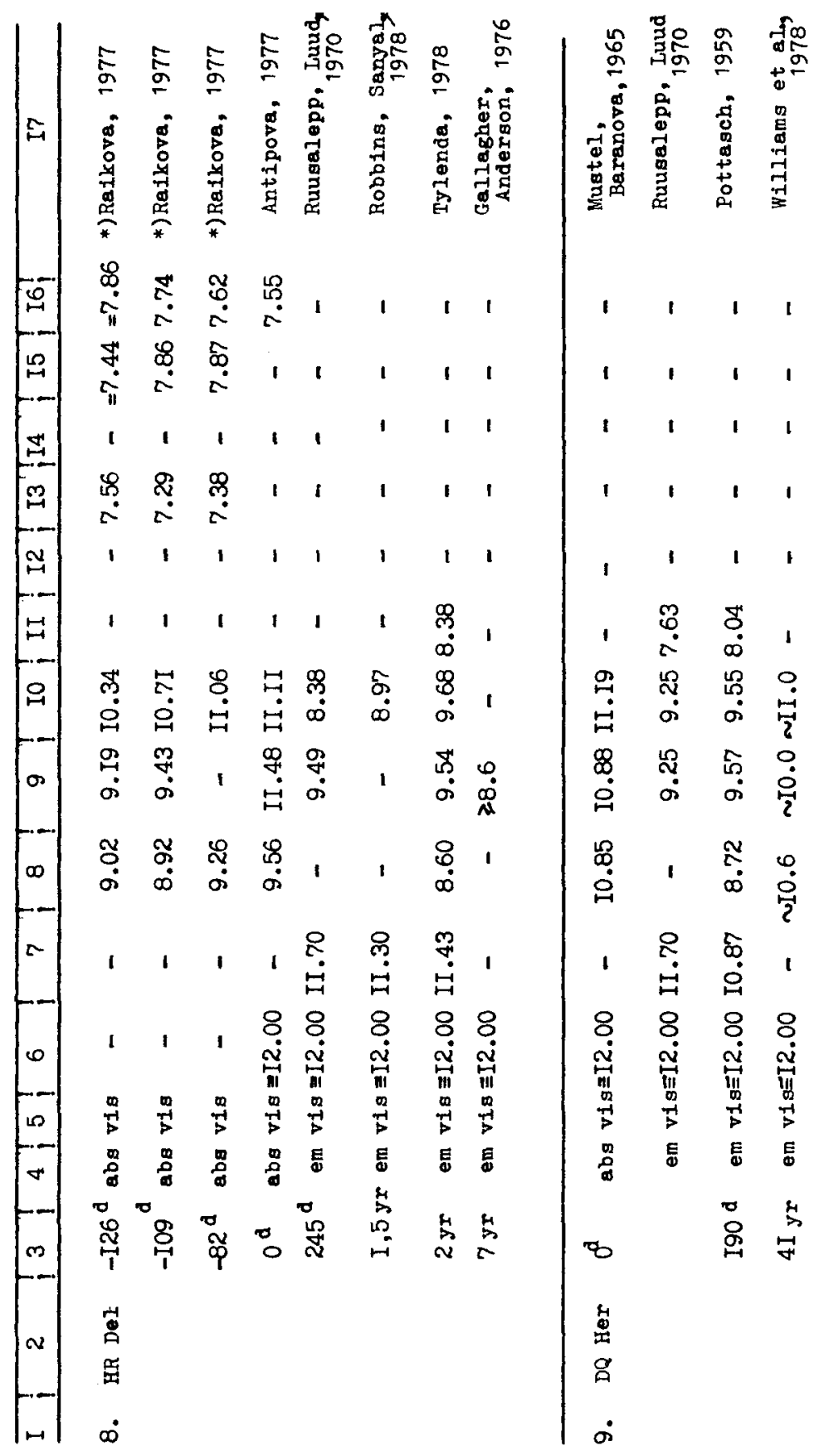




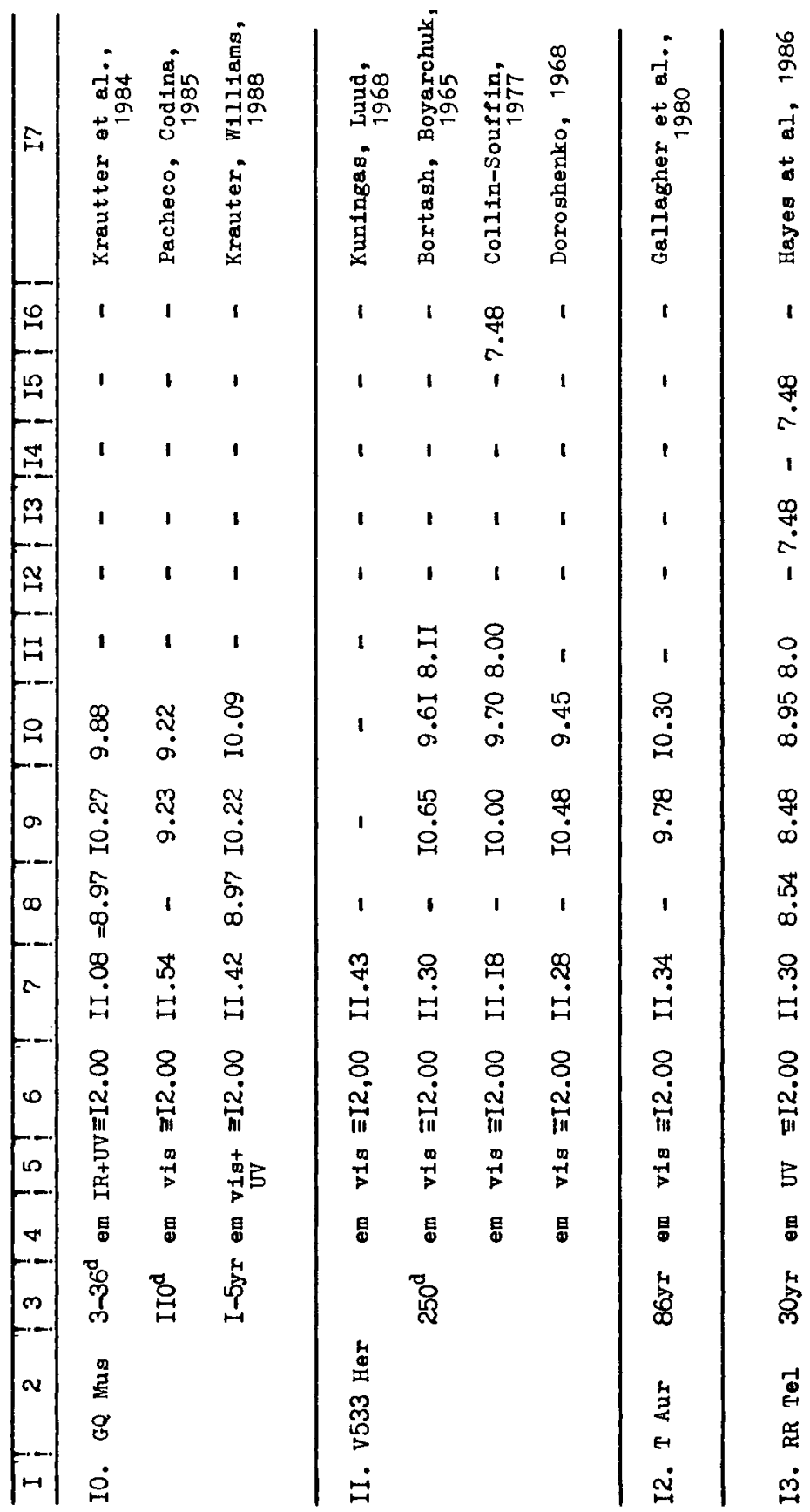




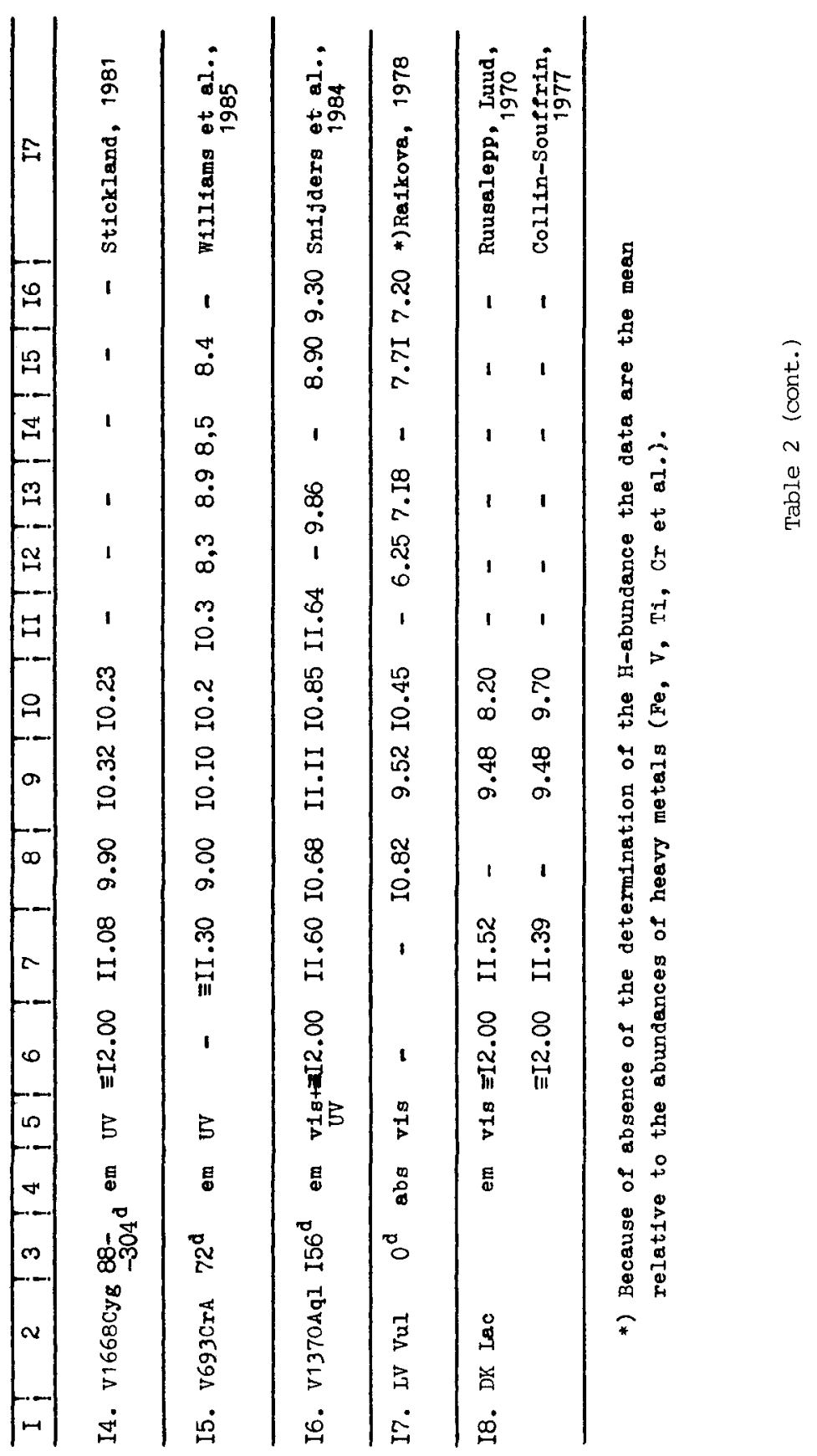




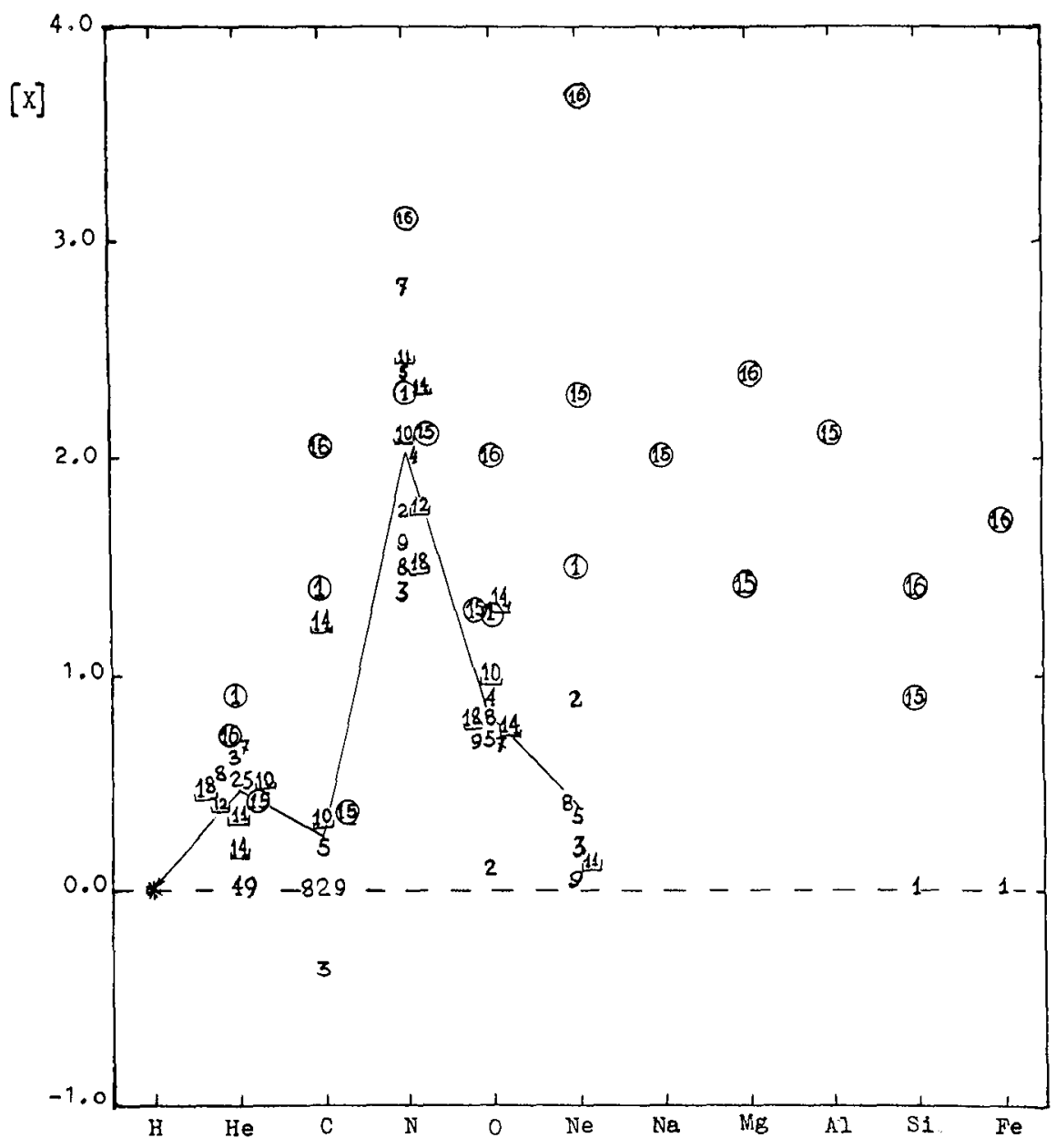

Fig. 1 Abundance anomalies of novae. Points are numbered following the object numbers given in Table 1. Positions in the diagram corresponding to two objects are labeled as l. Circles denote "neon" novae. 
accretion layer before the beginning of thermonuclear reactions. First of all, this affects the velocity class of the nova. Calculations based on the model with accreted matter enriched in CNO nuclei provide light curves and outflow velocities which are typical for a fast nova.

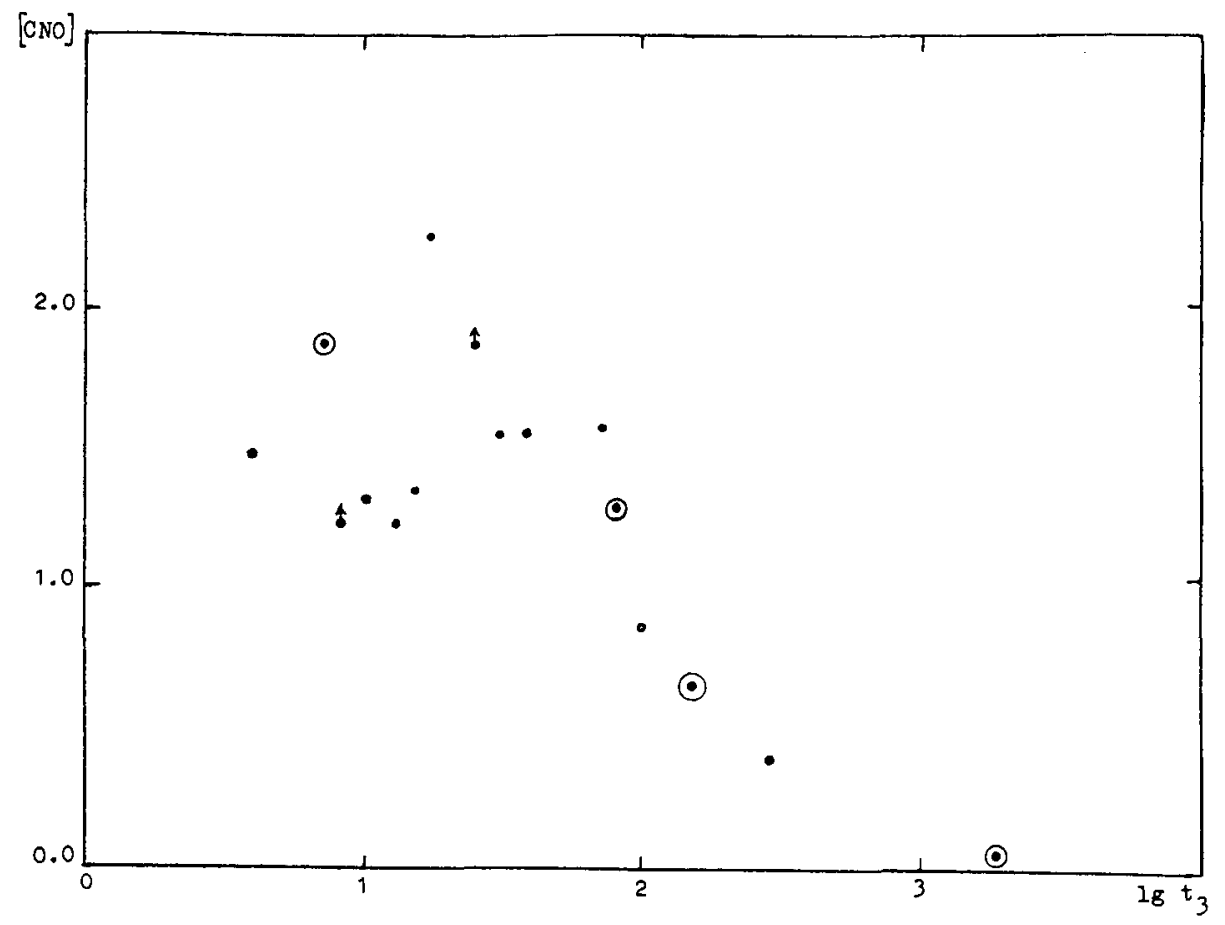

Fig. 2 Dependence of the sum of CNO-nuclei on the velocity class of novae.

On the contrary, a white dwarf with solar chemical composition of the accreted matter is a good model for an outburst of a very slow nova such as, for example, HR Del (Sparks et al. 1978). Thus, a correlation of the abundance of CNO-elements with the velocity class of the nova should be observed.

The first observations showing a correlation between the abundance of $\mathrm{N}$ in the nova envelopes and $t_{3}$ ( $t$ ime needed for the nova to decrease three magnitudes from maximum) was published by Ruusalepp and Luud (1970). Later, using determinations of chemical composition in the nova envelopes based on optical observations, Pacheco and Codina (1985) showed that there is less oxygen in the envelopes of novae with smaller $t_{3}$. The data obtained on the basis of ultraviolet spectra confirm this result (Cassatella and Selvelli 1988). Taking into account that, according to the theory of nucleosynthesis during thermonuclear runaway, the total number of atoms of the elements $C, N$ 

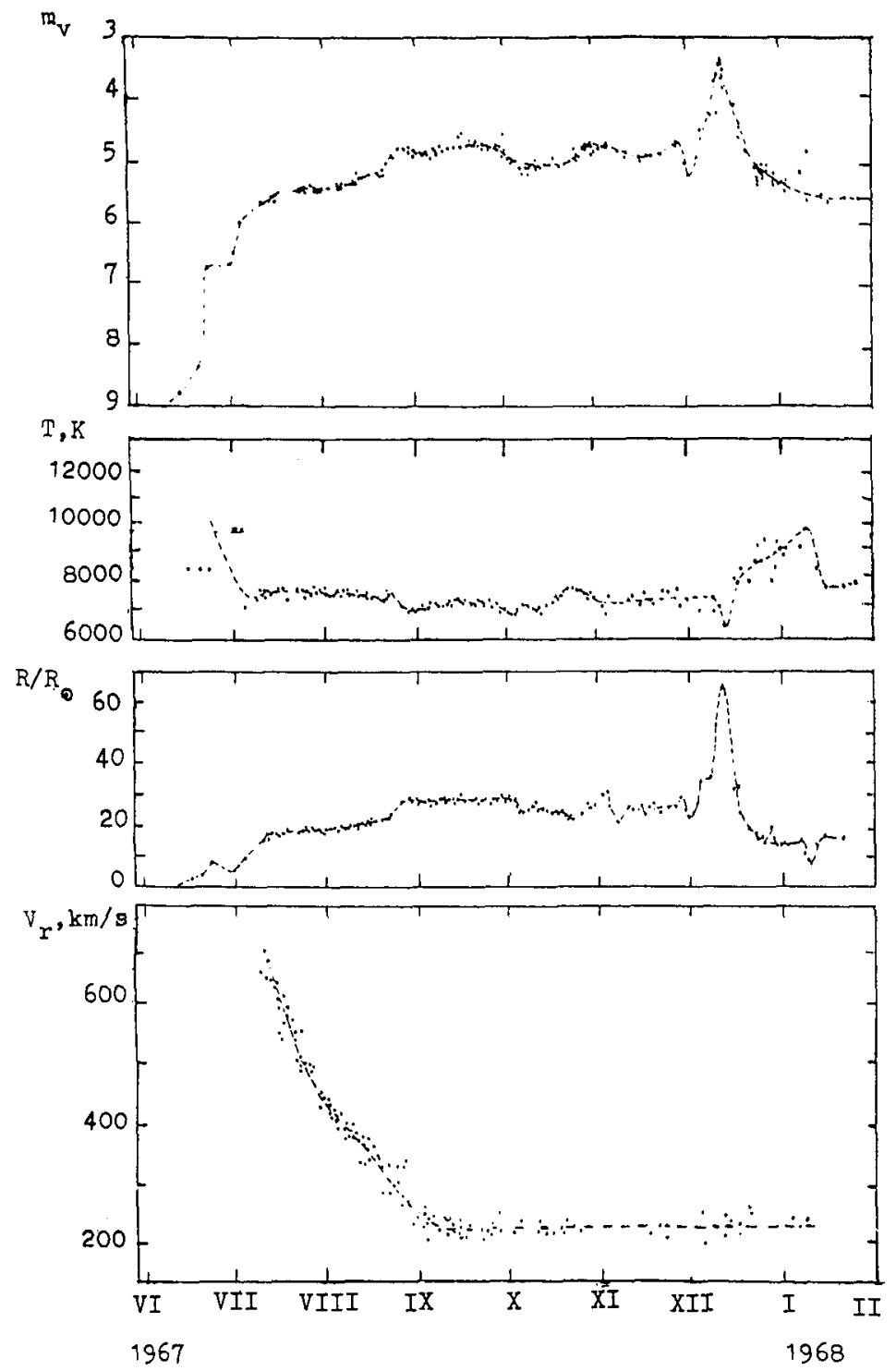

Fig. 3 Changes of brightness, temperature, radius and velocity of the ejected matter for the nova HR Del during the pre-maximum period. 
and $O$ must remain the same, it seems useful to analyse the correlation of the sum of the CNO nuclei with the parameter $t_{3}$. For novae with more than one determination of the chemical composition, the data obtained as late as possible after the outburst must be chosen since during the earlier stages of the outburst stratification effects of the physical conditions in the envelope are not negligible. A diagram showing the dependence of the sum of CNO nuclei on the parameter $t_{3}$ is presented in Fig. 2. There are several post-novae which were observed for determinations of chemical abundances many years after the outburgt, when their envelopes were certainly optically thin and more homogeneous than at the initial stages. Such determinations should be considered as the most reliable. In Fig. 2 these stars are marked with a circle. One may conclude that the correlation of $\Sigma$ CNO with the velocity class of novae exists indeed.

Since during the thermonuclear transformations the total number of CNO nuclei must remain constant, the following model was proposed to explain their overabundance in the burning zone (Colvin et al. 1977): the outburst occurs on the surface of a carbon-oxygen white dwarf; carbon and oxygen come into the lowest part of the accretion layer as the result of convective mixing with the outward parts of the carbon-oxygen white dwarf. The analysis of the chemical composition of HR Del during its pre-maximum stage possibly confirms this model. HR Del is a very slow nova which is observed to have a long (about half a year) pre-maximum period, during which its brightness, temperature and radius were practically constant, and the radial velocities of the absorption lines shoved a continuous outflow of matter from the nova (dynamical equilibrium was taking place), gee Fig. 3. Using the curve-of-growth method, the chemical composition of the nova was determined on the basis of the absorption lines at three different epochs during the pre-maximum period (Raikova 1977) and at maximum brightness (Antipova 1977). The abundances of $C$, $N$ and $O$ at the different dates are shown in Fig. 4. One can see from the figure that there is a tendency for the abundance of these elements to increase with time. Since a relatively thin shell layer is observed in the absorption lines "reversing" layer above the "pseudo-photosphere"), under the conditions of dynamical equilibrium, matter from deeper layers flowg through the reversing layer. The increase of the abundances of $C, N$ and $O$ with $t i m e$ may then be regarded as a confirmation that the nuclei of these elements get in this layer from the white dwarf.

Thus, the analysis of both the absorption and the emission lines lead to the conclusion of abnormally high abundances of $C$, $N$ and $O$ nuclei in the nova shells. The differences in the absolute values of the anomalies must obviously be connected with the above mentioned inhomogeneities in the chemical composition of nova shells: the absorption lines arise from a thin layer of the shell and denote its chemical composition; the emission lines are formed in a much broader layer of the shell, so that the derived abundances are to be regarded as average values in the shell and consequently may result lower than those obtained on the basis of the absorption spectrum at maximum.

He-abundance. In the review by Collin-Souffrin (1977) the value [He] = 0.25 was determined as mean value for several novae and the supposition was made that faster novae contain more helium than slower novae. 

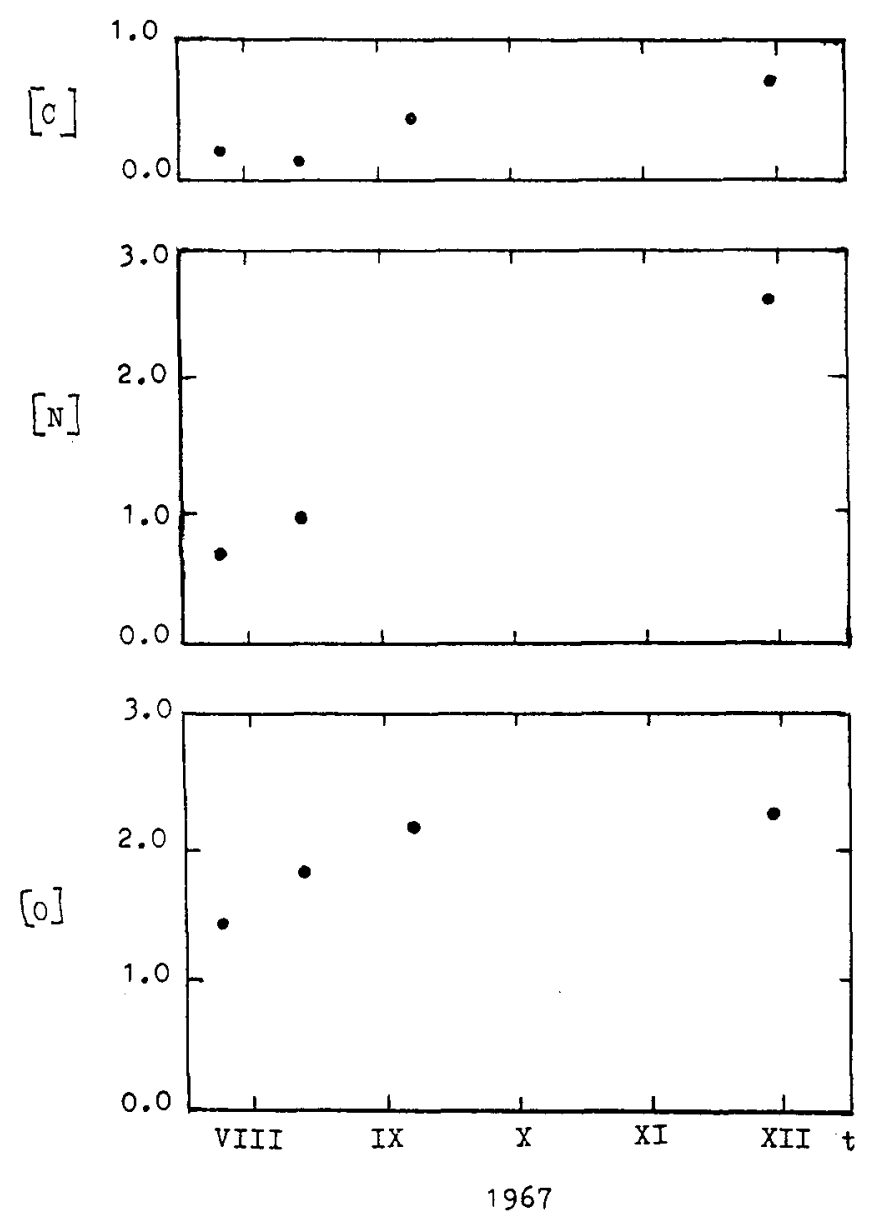

Fig. 4 Abundances of $\mathrm{C}, \mathrm{N}$ and $\mathrm{O}$ at various dates during the pre-outburst period of the nova HR Del. 
Using the data of Table 2, we have plotted in Fig. 5 the values of [He] vs. $t_{3}$. One can see that there is no correlation between the overabundance of $\mathrm{He}$ and the velocity class of novae. On average, the He abundance in novae is slightly higher than "normal"; the mean value [He] $=0.40$ is close to the value given by Collin-Souffrin (1977).

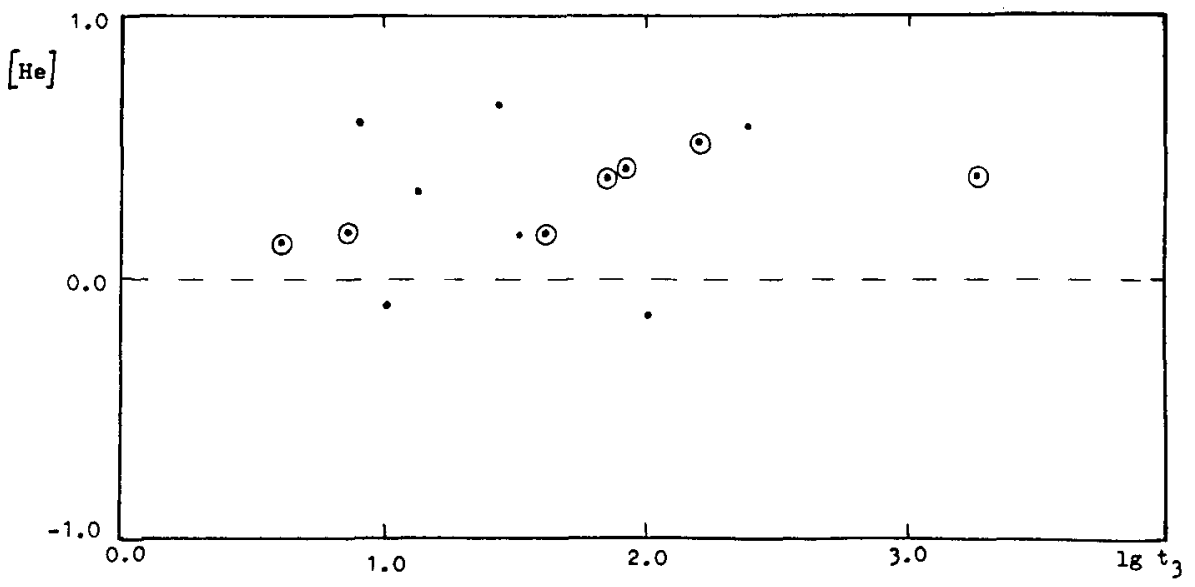

Fig. 5. Helium abundances in the envelopes of novae with different velocity clasges.

Critically analysing the methods of determination of the Helium abundance in novae envelopes, Ferland (1978) mentions at least two reasons which lead to an overestimation of the Helium abundance. Firstly, the chemical composition is often determined before the effects of self-absorption in the Balmer lines, used in the analysis, have become negligible. The pregence of self-absorption causes a change of the intensity of the hydrogen lines and, therefore, leads to wrong values of the relative abundance of Helium. Secondly, one must bear in mind that triplets lines may also give wrong values of the Helium abundance as compared with the values based on singlet lines because of the metastability of level $2^{3} \mathrm{~S}$. Taking into account these two circumstances and having made the necessary corrections, Ferland (1978) obtained a "normal" abundance of Helium in V1500 Cyg and draw a conclusion about the solar Helium abundance to be obviously typical also for other novae. However, he also assumed that it is possible that novae may not have all one and the same abundance of Helium, and that this could be easily checked by analysing old novae with optically thin envelopes. By now several of such investigations have already been performed (Williams 1979; Williams et al. 1978; Williams 1982; Ferland and Shields 1978; Gallagher et al. 1980). The circles in Fig. 5 indicate the Helium abundance obtained in the above papers as well as the Helium abundance from Ferland and Shields (1978) and Bartash and Boyarchuck (1965), in which the self-absorption in the Balmer lines was corrected for. It can be seen from the figure that different novae can indeed have different amounts of Helium. Since no correlation is observed between He and CNO abundances in the nova 
envelopes, then the source of the CNO overabundances must have little influence on the Helium abundance (Ferland and Shields 1978). According to the assumption in Colvin et al. (1977), much of $C$ and $O$ result from convective mixing of the upper layers of the nucleus of the white dwarf with the accretion matter, while the abundances of He and Fe correspond to the chemical composition of the cool component. In this case, if the correlation in Fig. 5, based on the best determinations of the Helium abundance, is real, it could mean that the cold componenta of slow novae are more evolved than those of fast novae.

Ne-abundance. According to Table 2 and Fig. 1, a number of stars show an overabundance of Neon. Among them there are both fast novae (like V150O Cyg) and slow novae (like RR Pic). No correlations were found though there are very little determinations of the Neon abundance. The overabundance of $\mathrm{Ne}$ in the envelopes of novae may be real; in this case, since it is not formed in the process of the thermal nuclear synthesis during the nova outburst it must come in the envelope from a white dwarf nucleus together with $C$ and $O$ (Ferland and Shields 1978). However, one should bear in mind also the following. Very often, the spectra of novae contain lines of very different stages of ionization, whose intensities cannot be explained by one source of ionization only - a central star emitting blackbody radiation. So, the analysis of the envelope HR Del has shown that the only way to remove this difference is to assume the ionizing radiation to consist of two components with different temperatures (Tylenda 1978). The conclusions about the existence of both hot and cold regions in the nova envelopes were derived following the analygis of the novae both at early outburst stages (Tylenda 1978) and at very late stages, many years after the outburst (Williams 1982; Williams et al. 1978; Gallagher et al. 1980). In the spectra of novae one can observe lines from [Ne III], [Ne IV], [Ne V], whose potentials lie between 41 and $97 \mathrm{eV}$, values which, on average, are higher than the potentials for the lines of the various stages of ionization of $\mathrm{C}, \mathrm{N}$ and $O$, from which $T_{e}$ is usually estimated. Therefore, the Ne lines can also arise from regions which are different from those where the CNO lines are formed. Applying a value of lower $T_{e}$, obtained from the lines of CNO elements, to the Neon lines may lead to an overestimation of Neon abundance in the nova envelope. The problem of the abundance of Neon in novae is still to be solved.

Na, Mg, Al, Si, Fe-abundances. Abnormally high abundances of these elements are indicated only in two novae: V693 CrA and V1317 Aql (Williams et al. 1985; Snijders et al. 1984). Both investigations have been performed by analysing the UV spectra. It should be noted that the UV data were complemented by spectroscopic optical data. The range 1200-3300 A contains several lines of elements and ions that cannot be observed in the optical range. They can be used for the determination of the abundances of chemical elements. Many of the lines observed in UV range are resonance lines. While analysing such lines, one should remember that the exciting energy of upper levels in the UV range is high; for example, to transfer in $\lambda 1600 \mathrm{~A}$, the excited level energy is $E \sim h r \sim 8 \mathrm{eV}$, while for similar transitions in the optical spectral range (for example $\lambda 5000$ A) this value is approximately equal to $2.5 \mathrm{eV}$. It is clear that a small error in the temperature when determining the abundances of the elements from 
the lines in the UV range would cause greater errors in the egtimation of the number of atoms on the ground level (and therefore, of the element abundance), than that using spectra in the optical range.

In the analysis of the spectrum of v693 CrA (Williams et al. 1985), the absence of pairs of lines sensitive to $T$. in the UV range did not allow to obtain a good estimation of $T_{\text {, and in the }}$ calculation of the abundances of chemical elements the value T. $15000 \mathrm{~K}$ was adopted, typical for planetary nebulae. It should also be noted that the analysis was carried out during the period of the so called "quasicoronal" stage, when in the spectrum, besides the lines of the middle ionization stages (He II, O III], o IV], N III], C III]), there were also lines of the forbidden transitions of the highly ionized atoms [Na V], [Si IX], [AIVIII], [Mg VII], etc., most of which are observed in the spectrum of the Solar corona. The presence of coronal lines in the spectra of novae is explained by the existence in the nova envelope of regions of very hot gas, where these lines are formed (Ferland et al. 1977; Andrillat and Collin-Souffrin 1977). Therefore, for the determination of chemical abundances from these lines one should use the temperature value, appropriate for these particular regions. If this is not taken into account, it would cause errors in the determination of the chemical composition, more in the UV range than in the optical range.

All the above can also be fully applied to the nova vi370 Aql (Snijders et al. 1984), in whose shell the abundances of any of the analyzed elements has not proved to be "normal". All of them (C, N, $O$, Ne, $\mathrm{Mg}, \mathrm{Al}, \mathrm{Si}, \mathrm{S}$ and $\mathrm{Fe}$ ) show overabundances by factors 30 to 3000 .

One can draw the following conclusions from the above review:

1. Both methods for the determination of the chemical composition of novae (from emission lines and from absorption lines) give the same results: the shells ejected by novae during outburst show abnormally high abundances of the elements $\mathrm{C}, \mathrm{N}, \mathrm{O}$ and He. Although these methods are applicable to different phases of the outburst (for example, absorption lines can be analysed near the maximum brightness, when the emission lines cannot be observed), they nevertheless supplement each other, allowing one to investigate on the changes of the chemical composition in novae during the development of the outburst.

2. It is important to study novae from spectra both in the UV range and in the optical ranges. The determination of the number of ions from lines in different stages of ionization including lines which can only be observed in the UV range, allows to obtain a higher accuracy on the abundances of the chemical elements in the ghells of novae.

3. To determine the chemical composition of the matter ejected during the nova outburst, it is worthwhile to observe the nova shells many years after the outburst, when their envelopes have become absolutely transparent in the lines and the lines are not distorted by self-absorption, and inhomogeneities in the shells, important at the initial stages, are now minimal. It can be seen from Fig. 1 that the determinations of the chemical composition of the shells available now, many years after the outburst, give smaller scatter than those performed at earlier stages of the outburst. 


\section{REFERENCES}

Andrillat, Y., Collin-Souffrin, S.: 1977, in "Novae and Related Stars", ed. M. Friedjung (Dordrecht: Reidel Publ. Co.), p. 137

Antipova, L.I.: 1977. Astron Z., 54, 68

Bortash, T.M., Boyarchuk, A.A.: 1965, Izv. Crimean Aph. Obs., $\underline{33}$, 173

Boyarchuk, A.A., Galkina, T.S., Gershberg, R.E., Krasnobabtsev, V.I., Rachkovskaya, T.M., Shaktovskaya, N.I.: 1977, Astr. Z., 54, 458

Cassatella, A., Selvelli, P.L.: 1988, in "A decade of UV Astronomy with IUE, ESA-SP 281, Vol. 1, p. 9

Collin-Souffrin, S.: 1977, in "Novae and related Stars", ed. M. Friedjung (Dordrecht: Reidel Publ. Co), p. 123

Colvin, J.W., van Horn, H.M., Starrfield, S.G., Truran, J.W.: 1977, Astrophys. J., 212, 791

Doroshenko, V. T.: 1968 , Astr. Z., 45, 121

Duerbeck, H.W.: 1981. Publ. Astron. Soc. Pac., 93, 165

Ferland, G.J.: 1978, Astrophys. J., 219, 589

Ferland, G.J., Shields, G.A.: 1978, Astrophys. J., 226, 172

Ferland, G.J., Lambert, D.L., Woodman, J.H.: 1977, Astrophys. J., 213, 132

Gallagher, J.S., Anderson, C.M.: 1976, Astrophys. J., 203, 625

Gallagher, J.S., Hege, E.K., Kopriva, D.A., Williams, R.E., Butcher, H.R.: 1980, Astrophys. J., 237, 55

Hayes, M.A., Nussbaumer, H.: $19 \overline{986}$, Astron. Astrophys., 161, 287

Krautter, J., Beuermann, K., Leitherer, C., Oliva, E., Moorwood, A.F.M., Deul, E., Wargau, W., Klare, G., Kohoutek, L., van Paradijs, J., Wolf, B.: 1984, Astron. Astrophys., 137, 307

Krautter, J., Williams, R.E.: 1988, The Messenger, 54,33

Kuningas, S., Luud, L.: 1968, Tartu Obs. Publ., 36,222

Lance, C.M., McColl, M.L., Uomoto, A.K.: 1988, Astrophys. J., Suppl. Ser. 66,151

Mclaughlin, D.B.: 1936, Astrophys. J., 84, 104

Mustel, E.R., Baranova, L.I.: 1965. Astr. Z., 42, 42

Mustel, E.R., Boyarchuk, M.E.: 1959, Izv. Crimean Aph. Obs., 21, 3

Mustel, E.R., Boyarchuk, M.E.: 1959a, Astr. Z., $\underline{36}, 762$

Pacheco, J.A. de Freitas: 1971, Mon. Not. R. astr. Soc., 181, 421

Pacheco, J.A. de Freitas, Codina, S.J.: 1985, Mon. Not. R. astr. Soc., 214,481

Pottasch, S.: 1959, Ann. d'Ap., 22, 412

Pottasch, S.: 1967, Bull. Astron. Inst. Neth., 19, 227

Raikova, D.V.: 1977, Astron. Z., 54, 55

Raikova, D.V.: 1978, Dissertation

Robbins, R.R., Sanya1, A.: 1978, Astrophys. J., 219, 985

Ruusalepp, M. , Luud, L.: 1970, Tartu Obs. Publ., $\underline{39}, 89$

Snijders, M.A.J., Batt, T.J., Seaton, M.J., Blades, J.C., Morton, D.G.: 1984, Mon. Not. R. astr. Soc., 211, L7

Sparks, W.M., Starrfield, S., Truran, J.W.: 1978, Astrophys. J., 220, 1063

Starrfield, S., Truran, J.W., Sparks, W.M.: 1977, in "CNO-Isotopes in Astrophysics", ed. J.Audouze, (D. Reidel, Dordrecht, Holland)

Starrfield, S., Truran, J.W., Sparks, W.M., Kutter, G.S.: 1972, Astrophys. J., 176,169

Stickland, D.J.: 1983, Astrophys. Space Sci., 92, 197

Stickland, D.J., Penn, C.J., Seaton, M.J., Snijders, M.A.J., Storey, P.J. : 1981, Mon. Not. R. astr. Soc., 197, 107 
Tylenda, R.: 1978, Acta Astron., 28, 333

Williams, R.E.: 1977, in "The Interaction of Variable Stars with their Environment", IAU Colloquium No. 42, Bamberg, Sept. 6-9, 1977, ed. R. Kippenhahn, J.Rahe, w.Strohmeier, p. 242

Williams, R.E.: 1979, Astrophys. J., $\underline{228}, 482$

Williams, R.E.: 1982, Astrophys. J., $\underline{261}, 170$

Williams, R.E., Hey, E.P., Sparks, W.M., Starrfield, s.G., Wyskoff, S., Truran, J.W.: 1985, Mon. Not. R. astr. Soc., 212, 753

Williams, R.E., Woolf, N.J., Hege, E.K., Moore, R.L., Kopriva, D.A.: 1978, Astrophys. J., 224, 171 